\title{
Lagrangian condensation microphysics with Twomey CCN activation
}

\author{
Wojciech W. Grabowski ${ }^{1,2}$, Piotr Dziekan ${ }^{2}$, and Hanna Pawlowska ${ }^{2}$ \\ ${ }^{1}$ National Center for Atmospheric Research, Boulder, CO, USA \\ ${ }^{2}$ Institute of Geophysics, Faculty of Physics, University of Warsaw, Warsaw, Poland
}

Correspondence: Wojciech W. Grabowski (grabow@ucar.edu)

Received: 28 August 2017 - Discussion started: 6 September 2017

Revised: 24 November 2017 - Accepted: 25 November 2017 - Published: 12 January 2018

\begin{abstract}
We report the development of a novel Lagrangian microphysics methodology for simulations of warm icefree clouds. The approach applies the traditional Eulerian method for the momentum and continuous thermodynamic fields such as the temperature and water vapor mixing ratio, and uses Lagrangian "super-droplets" to represent condensed phase such as cloud droplets and drizzle or rain drops. In other applications of the Lagrangian warm-rain microphysics, the super-droplets outside clouds represent unactivated cloud condensation nuclei $(\mathrm{CCN})$ that become activated upon entering a cloud and can further grow through diffusional and collisional processes. The original methodology allows for the detailed study of not only effects of CCN on cloud microphysics and dynamics, but also $\mathrm{CCN}$ processing by a cloud. However, when cloud processing is not of interest, a simpler and computationally more efficient approach can be used with super-droplets forming only when $\mathrm{CCN}$ is activated and no super-droplet existing outside a cloud. This is possible by applying the Twomey activation scheme where the local supersaturation dictates the concentration of cloud droplets that need to be present inside a cloudy volume, as typically used in Eulerian bin microphysics schemes. Since a cloud volume is a small fraction of the computational domain volume, the Twomey super-droplets provide significant computational advantage when compared to the original superdroplet methodology. Additional advantage comes from significantly longer time steps that can be used when modeling of $\mathrm{CCN}$ deliquescence is avoided. Moreover, other formulation of the droplet activation can be applied in case of low vertical resolution of the host model, for instance, linking the concentration of activated cloud droplets to the local updraft speed.
\end{abstract}

This paper discusses the development and testing of the Twomey super-droplet methodology, focusing on the activation and diffusional growth. Details of the activation implementation, transport of super-droplets in the physical space, and the coupling between super-droplets and the Eulerian temperature and water vapor field are discussed in detail. Some of these are relevant to the original superdroplet methodology as well and to the ice phase modeling using the Lagrangian approach. As a computational example, the scheme is applied to an idealized moist thermal rising in a stratified environment, with the original superdroplet methodology providing a benchmark to which the new scheme is compared.

\section{Introduction}

Traditional cloud modeling methodologies apply a continuous medium approach for all thermodynamic variables, that is, not only for the temperature and water vapor, but also for all forms of cloud condensate and precipitation. Such methodologies have been the workhorse of the cloudscale modeling from its early days (e.g., Kessler, 1963; Liu and Orville, 1969; Murray, 1970; Schlesinger, 1973; Klemp and Wilhelmson, 1978; Clark, 1979), but also in numerical weather prediction using global as well as limited-area models and in climate simulation. Since the edge of an icefree cloud represents a sharp transition from droplet-laden air close to saturation to unsaturated droplet-free air outside the cloud, numerical diffusion and dispersion errors impose stringent constraints on numerical schemes suitable for cloud modeling. For instance, since cloud and precipitation vari- 
ables are positive definite, any numerical scheme that introduces negative values to the numerical solution (e.g., during advection in the physical space) is not suitable for cloud simulation. Moreover, difficulties in representing sharp cloud edge discontinuities in thermodynamic fields are well appreciated by the cloud-scale modeling community, especially from the point of view of the supersaturation field, the key variable for the formation and growth of water and ice cloud particles (e.g., Grabowski and Morrison, 2008, and references therein).

The last couple of decades witnessed an increased interest in cloud-scale computational approaches that limit the abovementioned problems and attempt to better represent the truly multiphase nature of clouds. Among those, the particle-based Lagrangian method, referred to as the Lagrangian cloud model (Andrejczuk et al., 2008, 2010) or the "super-droplet method" (Shima et al., 2009), is of particular relevance (see also Riechelmann et al., 2012; Arabas et al., 2015; Hoffmann et al., 2015, among others). By representing formation and growth of natural cloud particles using a subset of those particles ("super-particles"), many problems haunting traditional Eulerian approaches are either eliminated or significantly reduced. For instance, formation of cloud droplets through activation of cloud condensation nuclei $(\mathrm{CCN})$ can be formulated in a straightforward way, and processing of $\mathrm{CCN}$ through collision-coalescence or chemical reactions within droplets can be simulated from first principles. In the continuous medium approach, however, these processes require either extreme computational effort (i.e., multidimensional bin schemes) or are simply impossible to consider without additional simplifications. In the Lagrangian approach for warm (ice-free) clouds, each superdroplet (SD hereafter) carries a set of attributes, such as the CCN size and composition, wet particle mass and multiplicity parameter (the latter being the number of real droplets each super-droplet represents), that allow the representation of condensation and associated latent heat release as well as the development of drizzle and rain. In previous applications of such a methodology, the super-droplets outside clouds represent unactivated CCN (haze) particles that become activated upon entering a cloud and can further grow through diffusional and collisional processes. Since the information about the $\mathrm{CCN}$ is available for each super-droplet, the methodology allows for detailed study of not only effects of $\mathrm{CCN}$ on cloud microphysics and dynamics, but also CCN processing by a cloud. However, when cloud processing is of no interest, the Twomey activation (Twomey, 1959) can be used with super-droplets forming when $\mathrm{CCN}$ is activated and no super-droplet existing outside a cloud as often applied in Eulerian bin microphysics models (e.g., Grabowski et al., 2011). Since cloud volume is a small fraction of the computational domain volume, the Twomey super-droplets allow significant savings when compared to CCN-based Lagrangian methodology. Moreover, significantly longer time steps can be used because modeling of $\mathrm{CCN}$ deliquescence is avoided.

This paper discusses the development and testing of a novel Lagrangian approach focusing on activation and diffusional growth of cloud droplets. Our motivation is to use this methodology to study the impact of turbulence and entrainment on the spectrum of cloud droplets in shallow warm boundary layer clouds, such as tropical or subtropical cumulus and subtropical stratocumulus (see idealized adiabatic parcel simulations discussed in Grabowski and Abade, 2017, hereafter GA17). The key aspect, difficult if not impossible to apply in the Eulerian approach, is the possibility to formulate a subgrid-scale statistical scheme and apply it to individual droplets taking advantage of a stochastic formulation along the Lagrangian particle trajectory as in GA17. The developments discussed here exclude collision-coalescence as only marginally relevant to the spectral broadening problem. Collision-coalescence can be included in a relatively straightforward way (see a review and tests of various approaches discussed in Unterstrasser et al., 2017) and adding it to the model described here will be pursued in the future.

The next section presents analytic formulation of the Twomey super-droplet scheme and discusses its implementation in the Eulerian fluid flow model. The specific aspects discussed in detail are the treatment of the activation on the finite-difference fluid flow model grid, transport of super-droplets across the Eulerian grid, and coupling between the super-droplets and Eulerian thermodynamics. Section 3 presents examples of model simulations where the Lagrangian thermodynamics is included in an anelastic smallscale fluid flow model and applied in moist rising thermal simulations. A traditional super-droplet scheme (i.e., following $\mathrm{CCN}$ particles and allowing their activation and growth of resulting cloud droplets) is used to show consistency between the two methods. Brief conclusions and the outlook are presented in Sect. 4.

\section{Formulation}

\subsection{Analytic formulation}

Model equations describe evolution in space and time of the potential temperature, water vapor mixing ratio, and a set of Lagrangian point particles representing activated cloud droplets. The potential temperature $\Theta$ and water vapor mixing ratio $q_{\mathrm{v}}$ equations are as follows:

$$
\begin{aligned}
& \frac{\mathrm{D} \Theta}{\mathrm{D} t}=\frac{L_{\mathrm{v}}}{c_{\mathrm{p}} \Pi} C_{\mathrm{d}}, \\
& \frac{\mathrm{D} q_{\mathrm{v}}}{\mathrm{D} t}=-C_{\mathrm{d}},
\end{aligned}
$$

where $\mathrm{D} / \mathrm{D} t=\partial / \partial t+(\boldsymbol{u} \cdot \nabla)$ is the material (advective) derivative, $C_{\mathrm{d}}$ is the condensation rate, $\Pi=\left(p / p_{0}\right)^{R / c_{\mathrm{p}}}$ is the Exner function ( $p$ is the local pressure that in the anelas- 
tic system comes from the environmental profiles and $p_{0}=$ $1000 \mathrm{hPa}$ ), and $L_{\mathrm{v}}$ and $c_{\mathrm{p}}$ are the latent heat of vaporization and air specific heat at constant pressure, respectively. The condensation rate $C_{\mathrm{d}}$ is defined as the rate of change of the mass of cloud droplets. For the finite-difference model considered here, it can be calculated from the rate of change of mass of all cloud droplets located within a given grid cell:

$C_{\mathrm{d}}=\frac{\mathrm{d}}{\mathrm{d} t}\left(\sum_{i=1}^{N} \frac{4 \rho_{\mathrm{w}}}{3 \rho_{\mathrm{a}}} \pi r_{i}^{3} N_{i}\right)$

where $\rho_{\mathrm{w}}$ and $\rho_{\mathrm{a}}$ are the water and air density, respectively, and $r_{i}$ and $N_{i}$ are the radius and concentration of $N$ cloud droplet classes (bins) into which all droplets located within the grid cell are grouped. Such a definition has some similarity to the way condensation rate is calculated in Eulerian bin microphysics schemes, an analogy that will be useful when droplet activation is discussed later in this section. Given the supersaturation $S=q_{\mathrm{v}} / q_{\mathrm{vs}}-1$ (where $q_{\mathrm{vs}}$ is the saturated water vapor mixing ratio) the individual droplet growth equation is as follows:

$$
\frac{\mathrm{d} r_{i}}{\mathrm{~d} t}=\frac{A S}{r_{i}+r_{0}}, \quad \text { where } A=\frac{q_{\mathrm{vs}} \rho_{\mathrm{a}} D_{\mathrm{v}}}{\rho_{\mathrm{w}}\left(1+\frac{L_{\mathrm{v}}}{c_{\mathrm{p}}} \frac{\partial q_{\mathrm{vs}}}{\partial T}\right)},
$$

$r_{0}=1.86 \mu \mathrm{m}$ is a parameter that allows including kinetic effects (e.g., Clark, 1974; Kogan, 1991), and $D_{\mathrm{v}}$ is the diffusivity of water vapor in the air that depends on the temperature and pressure. A convenient feature of Eq. (4) is that the rate of growth remains bounded when $r_{i}$ approaches zero. The coefficient $A$ used in Eq. (4) is an approximate form of a more general formulation as given, for instance, by Eq. (3) in Grabowski et al. (2011). The approximate formulation (Eq. 4) can be obtained by assuming that the thermal conductivity of air $K$ is approximately given by $K=c_{\mathrm{p}} \rho_{\mathrm{a}} D_{\mathrm{v}}$, that is, replacing thermal diffusivity with the diffusivity of water vapor (this is accurate to about 10-15\%). Note that Grabowski et al. (2011) and GA17 applied a constant value $A=0.9152 \times 10^{-10} \mathrm{~m}^{2} \mathrm{~s}^{-1}$. Droplets are carried by the airflow (i.e., droplet sedimentation is excluded), an assumption justifiable by the exclusion of droplet collisions, the spatial scales considered (tens of meters and larger), and the length of simulations (up to a few tens of minutes). Thus, the evolution of the $i$ th droplet position $\boldsymbol{x}_{i}$ is calculated as

$$
\frac{\mathrm{d} \boldsymbol{x}_{i}}{\mathrm{~d} t}=\boldsymbol{u}\left(\boldsymbol{x}_{i}, t\right)
$$

where $\boldsymbol{u}$ is the air flow velocity predicted by the dynamical model.

Considering typical cloud droplet concentrations in natural clouds, from several tens to a few thousands per cubic centimeter, it is computationally impossible to follow all cloud droplets in the entire volume of even a very small cloud. Thus, the Lagrangian methodology involves following only a selected (typically relatively small) subset of cloud droplets, referred to as super-droplets following Shima et al. (2009). This is again in the spirit of using a finite (and typically relatively small) number of classes (bins) in the Eulerian bin microphysics scheme. As in Andrejczuk et al. (2008), Andrejczuk et al. (2010), Shima et al. (2009), and Riechelmann et al. (2012), among others, the list of attributes for each super-droplet includes the position $\boldsymbol{x}_{i}$, radius $r_{i}$, and multiplicity. The latter depicts the number of particles represented by a single super-droplet. Other attributes can be added if needed, for instance, the local supersaturation perturbation (on top of the grid-scale supersaturation predicted by the flow model) that can affect super-droplet growth in Eq. (4) as in GA17 or the subgrid-scale velocity perturbation that can affect the motion of the super-droplet in Eq. (5).

\subsection{Numerical implementation}

As will be discussed in Sect. 3, the novel super-droplet scheme has been included in the finite-difference anelastic model EULAG and its simplified version referred to as babyEULAG. EULAG and babyEULAG apply nonoscillatory-forward-in-time (NFT) integration scheme (e.g., Smolarkiewicz and Margolin, 1993; Grabowski and Smolarkiewicz, 2002; Prusa et al., 2008). For the coupling with super-droplets, the NFT scheme for the potential temperature (Eq. 1) and water vapor mixing ratio (Eq. 2) has been modified to include the Euler-forward time integration, that is,

$\Psi(t+\Delta t)=[\Psi(t)+F(t) \Delta t]_{0}$,

where $\Psi$ is either $\Theta$ or $q_{\mathrm{v}}, F$ represents the right-handside of Eqs. (1) and (2), and subscript " 0 " depicts the departure point of the fluid trajectory. This is the same as applied in the bin microphysics versions of EULAG in Wyszogrodzki et al. (2011, Sect. 2.2 therein) and babyEULAG in Grabowski and Jarecka (2015, appendix therein). Exploring the analogy between Lagrangian (trajectory-wise) and Eulerian (control-volume-wise) description of the fluid flow equations, Eq. (6) is solved using the flux-form monotone advection scheme MPDATA (e.g., Smolarkiewicz, 2006). Thus, the second-order-in-space and centered-in-time advection scheme is combined with the first-order-in-time (Euler forward) integration of the forcing term. A similar approach is used for the super-droplets, where the super-droplet transport is computed using the predictor-corrector scheme and droplet growth is calculated using the first-order-in-time uncentered scheme. It should be stressed the momentum equation in the host babyEULAG and EULAG models is advanced applying the centered in time scheme.

\subsection{Super-droplet initiation}

The key element of the scheme presented here that makes it distinct from the approach used in Andrejczuk et al. (2008, 2010), Shima et al. (2009), Riechelmann et al. (2012), 
Arabas et al. (2015), and others, is the way super-droplets are created. The original implementations assume that superdroplets fill the entire computational domain, and they initially represent deliquesced (humidified) $\mathrm{CCN}$ in equilibrium with their local environment. These unactivated superdroplets may become activated if environmental conditions dictate so, for instance, when passing through the cloud base. When CCN dry radius is one of the super-droplet attributes, the original approach allows explicit representation of aerosol processing by a cloud when collision-coalescence takes place (Shima et al., 2009, in which case the dry CCN after collision-coalescence combines dry $\mathrm{CCN}$ from colliding droplets), or when chemical reactions are included (e.g., Anna Jaruga; PhD dissertation, University of Warsaw). However, if neither of those processes is of interest, a significantly simpler approach can be used based on the so-called Twomey activation (Twomey, 1959) as often used in bin microphysics schemes (e.g., Grabowski et al., 2011). The Twomey approach links the number mixing ratio of activated CCN $N$ to the maximum supersaturation $S$ experienced by the cloudy volume. We will refer to the analytical or tabulated correspondence between $N$ and $S$ as the N-S relationship. Cloud base activation in the Eulerian bin microphysics scheme is simulated by introducing cloud droplets into appropriate bins until the supersaturation reaches its peak and activation is completed (see Grabowski et al., 2011). Without collisioncoalescence, the local droplet number mixing ratio provides information about the maximum supersaturation experienced by the volume in the past. With collision-coalescence, an additional model variable, the number mixing ratio of already activated $\mathrm{CCN}$, needs to be used to control whether additional CCN activation is required (see Sect. 2c in Morrison and Grabowski, 2008). The additional variable is also needed if a significant variability of the $\mathrm{CCN}$ exists in the computational domain (e.g., in the vertical direction) or if droplet sedimentation is included in the model physics.

The same approach can be used with super-droplets as already applied in GA17 in adiabatic parcel simulations. The key idea is that super-droplets are created in supersaturated conditions when the local concentration of activated droplets as given by the Twomey relationship is smaller than the one dictated by the local supersaturation. When a complete evaporation of cloud droplets occurs in subsaturated conditions, super-droplets are simply removed from the superdroplet list. Hence, no super-droplets exist outside of cloudy volumes, similarly to traditional Eulerian bin microphysics schemes. It follows that super-droplets with Twomey activation provide significant computational advantage over the traditional Lagrangian approach because only a relatively small number of super-droplets has to be used. Note that in the Eulerian bin scheme the computational expense of the droplet transport in the physical space is independent of whether droplets fill a small or a large fraction of the domain. This is because each bin needs to be advected separately in the physical space and the computational effort is indepen-

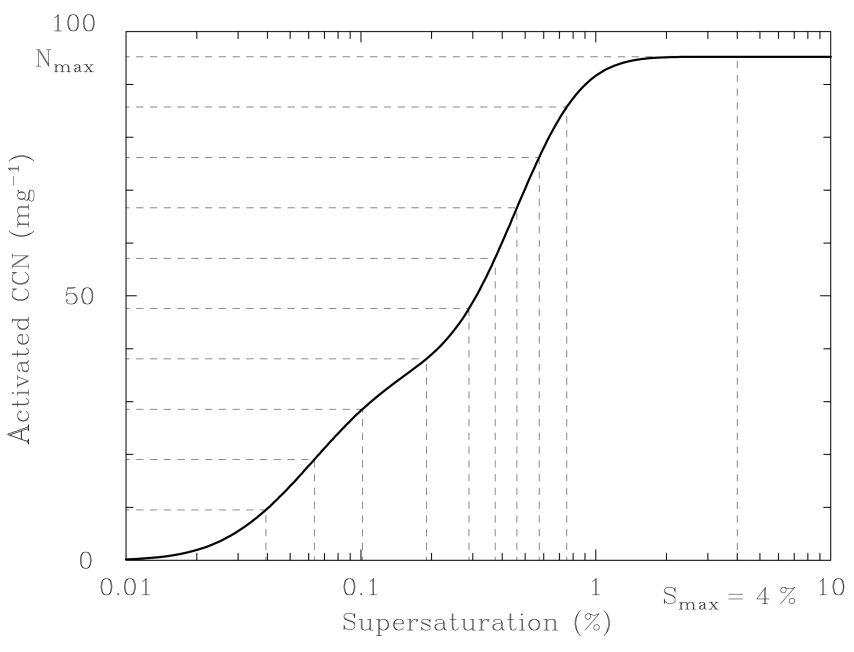

Figure 1. Thick line: number mixing ratio of activated $\mathrm{CCN}$ as a function of the supersaturation, the Twomey relationship, used in simulations described herein. Thin dashed lines illustrate numerical implementation of the CCN activation scheme. See text for details.

dent of whether the entire domain or just its small fraction is filled with droplets. It is worth pointing out that applying Twomey activation to create cloud droplets in the Lagrangian warm-rain thermodynamics bears similarities to the way ice particles are initiated in a particle-based Lagrangian model targeting ice processes (e.g., Sölch and Kärcher, 2010).

We assume the same CCN characteristics as in GA17 and Arabas et al. (2015). CCN characteristics include the chemical composition, the number mixing ratio of activated CCN for a given supersaturation (the Twomey relationship) and the activation radius. $\mathrm{CCN}$ are assumed to be composed of sodium chloride (sea salt; $\mathrm{NaCl}$ ). Idealized $\mathrm{CCN}$ distribution, the same as in Arabas et al. (2015), is represented by a sum of two lognormal distributions with number mixing ratio, mean radii, and geometric standard deviations (unitless) 57.33 and $38.22 \mathrm{~m} \mathrm{~g}^{-1}$ (i.e., per cubic centimeter for the air density of $1 \mathrm{~kg} \mathrm{~m}^{-3}$; these values come from converting the 60 and $40 \mathrm{~cm}^{-3}$ concentrations to the number mixing ratio using air density at the bottom of the computational domain in simulations discussed in Sect. 3), 20 and $75 \mathrm{~nm}$, and 1.4 and 1.6, respectively. The $\mathrm{N}-\mathrm{S}$ relationship is tabulated and the table is used as input to the super-droplet scheme. Once activated, the initial radius corresponding to the activation radius is assigned for each super-droplet. The latter is approximated as $8 \times 10^{-10} / S_{\text {act }}(\mathrm{m})$ as in GA17, where $S_{\text {act }}$ is the activation supersaturation; see Eq. (6) and Fig. 2 in Grabowski et al. (2011). In addition to the droplet radius, the model keeps track of the super-droplet multiplicity parameter (or attribute), that is, the number of droplets the super-droplet represents, Shima et al. (2009). A newly created super-droplet is placed randomly within a given grid cell and added to the super-droplet list. 


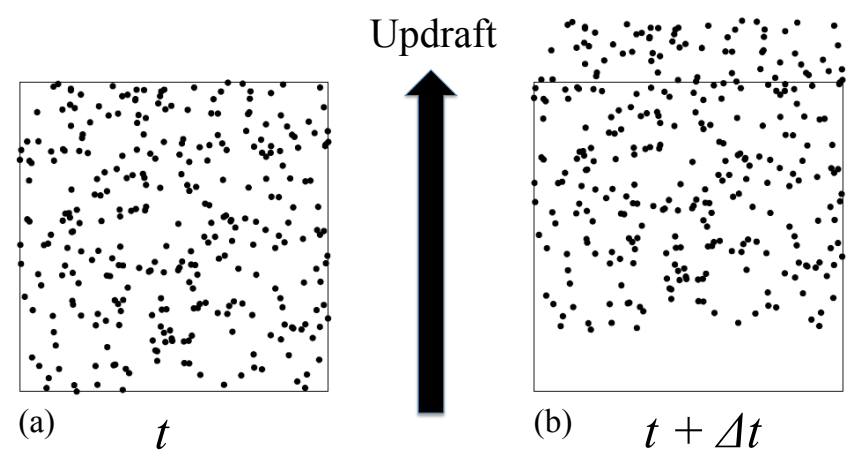

Figure 2. Illustration of the activation as represented on the fluid flow grid. Panel (a) shows locations of CCN activated at a given model time step. Panel (b) shows the situation at the next time step when activated $\mathrm{CCN}$ are advected away from the grid cell and activation of new $\mathrm{CCN}$ is required.

Figure 1, adopted from GA17, shows the N-S relationship and illustrates the way super-droplets are created. First, the maximum supersaturation $S_{\max }$ is selected. $S_{\max }$ has to exceed the maximum supersaturation anticipated in the simulation. $S_{\max }$ equal to $4 \%$ is used here as shown in Fig. 1. The corresponding maximum number mixing ratio of activated droplets $N_{\max }$ is divided by the number of droplet classes to be used in the simulations. The example in Fig. 1 assumes 10 classes whereas simulations typically apply several tens to several thousands of classes. New super-droplets are introduced to a given grid cell when the supersaturation predicted for that grid cell exceeds the supersaturation corresponding to the activation supersaturation of super-droplets already present in the grid cell. The approach illustrated in Fig. 1 ensures that the multiplicity parameter is the same for all super-droplets. This is beneficial because equal multiplicity minimizes statistical fluctuations of derived cloud quantities (such as the droplet concentration or liquid water content) when super-droplets are advected from one grid cell to another. The approach adopted here was suggested by simple one-dimensional advection tests completed during early stages of the scheme development. However, equal multiplicity is possibly the worst choice when collisioncoalescence is added to the scheme physics as pointed out by Unterstrasser et al. (2017). We note in passing that Unterstrasser and Sölch (2014) discuss various methodologies for introducing Lagrangian particles, including stochastic particle initiation as well as particle merging and splitting, that all aid computational efficiency of the Lagrangian cloud model. These need to be considered while expanding the scheme to include collision-coalescence.

When applied in a multidimensional fluid flow model, there is an additional issue with the proposed scheme that needs to be addressed. Figure 2 shows a single twodimensional grid cell at which formation of new superdroplets takes place at time $t$. At the next time step, $t+\Delta t$, super-droplets are advected upwards by the updraft, and a droplet-free volume is advected into the grid cell. Assuming that the supersaturation within the grid cell does not change, there is a need to activate new super-droplets as some of those present within the grid cell at the previous time step moved upwards. The new super-droplets should be introduced into the droplet-free volume (i.e., in the lower part of the grid cell in Fig. 2b) because unactivated CCN would be there. However, keeping track of volumes void of super-droplets during activation followed by advection is cumbersome. At the same time, adding new super-droplets randomly into the entire grid cell leads to the situation where super-droplets are not randomly distributed (i.e., more super-droplets is present in the upper part of the grid cell in Fig. 2). A simple approach adopted here is that all super-droplets are always randomly repositioned within a given grid cell once additional activation within that cell takes place.

\subsection{Transport of super-droplets in the physical space}

Super-droplets are advected in the physical space applying a predictor-corrector scheme to solve Eq. (5). The predictor step estimates the $n+1$ time level position from $n$ time level velocity as follows:

$\boldsymbol{x}_{\mathrm{p}}^{n+1}=\boldsymbol{x}^{n}+\boldsymbol{u}^{n}\left(\boldsymbol{x}^{n}\right) \Delta t$,

where the subscript " $p$ " depicts the predictor solution. The corrector step (subscript "c") is subsequently applied as follows:

$\boldsymbol{x}_{\mathrm{c}}^{n+1}=\boldsymbol{x}^{n}+\left[\boldsymbol{u}^{n+1}\left(\boldsymbol{x}_{\mathrm{p}}^{n+1}\right)+\boldsymbol{u}^{n}\left(\boldsymbol{x}^{n}\right)\right] \frac{\Delta t}{2}$.

The predictor-corrector scheme ensures the second-order accuracy for the time integration of the super-droplet transport. However, to increase accuracy, the corrector step can be repeated by replacing $\boldsymbol{x}_{\mathrm{p}}$ by the already-calculated $\boldsymbol{x}_{\mathrm{c}}$ in the $\boldsymbol{u}^{n+1}$ velocity on the right-hand side of Eq. (7). Note that velocity needs to be interpolated to the super-droplet position and repeating the corrector step increases the overall computational cost. We will test the benefit of the second correction step in the droplet advection procedure later in this section. It also needs to be pointed out that the super-droplet transport requires knowledge of the flow velocity at the $n+1$ time level in Eq. (8). Similarly to the case of EULAG's and babyEULAG's advection of the temperature and water vapor mixing ratio where advecting velocities need to be known at $n+1 / 2$ time level, the $n+1$ time level velocities in Eq. (8) are extrapolated from velocities available at $n-1$ and $n$ time levels.

Velocity interpolation to calculate super-droplet transport is the key element of the Lagrangian scheme. Since the EULAG model applies unstaggered grid (i.e., all variables are located at the same position), one possibility is to consider a grid cell whose four corners in two dimensions (eight vertices in three dimensions) form a rectangular (cuboid-shaped in three dimensions) grid cell. For a super-droplet located in such a grid cell, flow velocity at the droplet position can 


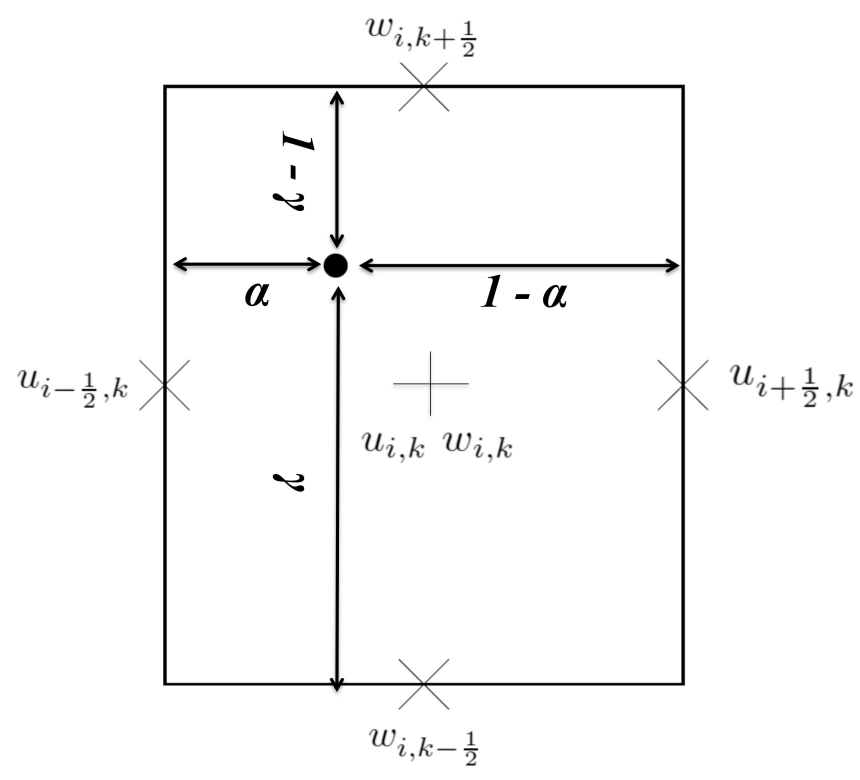

Figure 3. Illustration of the interpolation scheme used in the superdroplet transport scheme referred to as "simple" in the text. The rectangular box represents a single grid cell with $u$ and $w$ depicting horizontal and vertical velocities perpendicular to grid cell boundaries used in the advection scheme of the Eulerian model. The large dot represents droplet position.

be interpolated from the velocity values at the corners and vertices. Arguably the simplest possibility is to apply a bilinear (trilinear in three dimensions) interpolation scheme, but a more advanced scheme may be considered as well. However, the bilinear interpolation (and likely more advanced interpolation schemes) does not lead to physically consistent results as documented below.

Advection of the potential temperature and water vapor mixing ratio (as well as the velocity components) in EULAG is performed on the $\mathrm{C}$ grid (i.e., with the horizontalvertical velocities at the vertical-horizontal grid cell boundaries). Advective velocities come from interpolating velocity components predicted on the unstaggered grid into the $\mathrm{C}$ grid. Advective velocities satisfy the anelastic incompressibility condition $\nabla \cdot(\rho \boldsymbol{u})=0$, where $\rho(z)$ is the anelastic density profile. In two dimensions, the divergence of advecting velocities can be written in the finite-difference form as follows (see Fig. 3):

$$
\frac{u_{i+\frac{1}{2}, k}-u_{i-\frac{1}{2}, k}}{\Delta x}+\frac{w_{i, k+\frac{1}{2}}-w_{i, k-\frac{1}{2}}}{\Delta z}=-\frac{w}{\rho} \frac{\partial \rho}{\partial z},
$$

where the term on the right-hand side of Eq. (9) representing the change of the anelastic density with height is left in the analytic form as it is irrelevant to the discussion. With a single super-droplet located in the grid cell (see Fig. 3), the horizontal and vertical velocities can be interpolated using a simple scheme similar to that used in Arabas et al. (2015):

$u=\alpha u_{i+\frac{1}{2}, k}+(1-\alpha) u_{i-\frac{1}{2}, k}$,

$w=\gamma w_{i, k+\frac{1}{2}}+(1-\gamma) w_{i, k-\frac{1}{2}}$,

where $\alpha$ and $\gamma$ are nondimensional distances of the superdroplet position to the cell boundary as shown in Fig. 3. As documented in the Appendix A, such a definition ensures that the incompressibility condition (Eq. 9) is maintained on the subgrid scale of the grid cell. This, however, ensures that a deformation of the initially rectangular grid cell, as represented by passive advection of all passive particles initially located inside the cell, preserves the cell area (volume in three dimensions). We refer to the interpolation scheme (Eq. 10) as "simple" in the following discussion in contrast to the bilinear (or trilinear in three dimensions) interpolation scheme introduced previously.

To investigate the accuracy of the super-droplet transport scheme, a relatively simple test problem was designed. In the test, two-dimensional rising moist thermal simulations driven by the Eulerian bulk condensation scheme were used, applying the same simulation setup as in the super-droplet simulations (see Sect. 3.1). The predicted rising thermal flow (similar to the one shown later in the paper applying superdroplets) was applied to advect a large number of passive particles introduced to a fraction of the computational domain including the thermal and its immediate environment at the onset of the simulation. The number of passive particles varied from several tens to a few thousands per grid cell in various tests. In the rising thermal flow simulated by the model, one should expect the average number of particles per grid volume to slightly decrease because of the density decreasing with height. Moreover, the number should show statistical fluctuations due to advection of particles from one grid cell to another. The fluctuation amplitude should vary approximately as an inverse of the square root of the initial number of particles per grid cell. These assumptions provide the basis for evaluating the accuracy of the super-droplet transport.

Figure 4 shows evolution of the minimum and maximum number of passive particles per grid cell advected using the predictor-corrector scheme, with the upper and lower panels showing results from the bilinear and simple flow velocity interpolation, respectively. The extrema are calculated using only grid cells with the cloud water mixing larger than $0.01 \mathrm{~g} \mathrm{~kg}^{-1}$ (i.e., cloudy cells). Results are shown from simulations applying the predictor-only scheme (Eq. 7), the predictor-corrector scheme (Eqs. 7 and 8), and the predictorcorrector scheme with additional iteration of Eq. (8). The initial number of passive particles is either 100 (panels a and c) or 1000 (panels b and d) per grid cell. The standard deviation of the number of particles per grid cell after advection should be close to the square root of the initial number, that is, close to 10 and 30 particles (i.e., close to 10 and $3 \%$ of the particle number per grid cell) in the left and right panels, respec- 

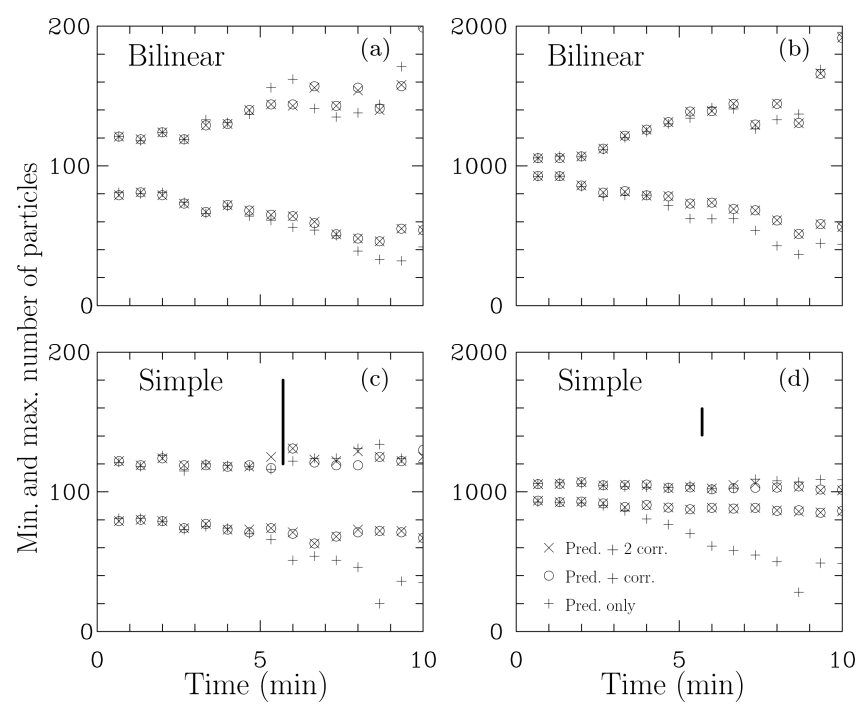

Figure 4. Evolution of the maximum and the minimum number of passive particles per grid cell in the bulk thermal simulations applying $(\mathbf{a}, \mathbf{b})$ the bilinear advection scheme and $(\mathbf{c}, \mathbf{d})$ the "simple" scheme (Fig. 3). Panels (a) and (c) show results applying 100 passive particle per grid cell, and (b) and (d) show results with 1000 passive particles per grid cell. Both schemes apply either the predictor step or the predictor-corrector with either one or two corrective iterations. The vertical bar length in (c) and (d) corresponds to six standard deviations of the expected number of particles per grid cell. See text for details.

tively. It follows that the difference between the maximum and minimum number of passive super-droplets per grid cell should be not significantly larger than a few standard deviations. As the upper panels of Fig. 4 show, the bilinear interpolation scheme leads to a larger difference starting around minute 2 of the simulations, and the difference increases with time. This is clearly unphysical as argued above. In contrast, the simple scheme with a single corrective iteration provides physically consistent results, that is, the difference between the maximum and minimum is several times the standard deviation of the initial particle number per grid cell, and such a difference is maintained throughout the $10 \mathrm{~min}$ long simulations. Only an insignificant improvement is simulated with the additional iteration of the corrective step (Eq. 8). Finally, a slight reduction of the mean concentration (located somewhere between the maximum and minimum symbols) is apparent in bottom panels of Fig. 4. This is because of the reduction of the droplet concentration due to the decrease of the air density with height (i.e., the term on the right-hand side of Eq. 9). It should be also pointed out that the difference between the predictor-only and the predictor-corrector schemes should decrease if a significantly shorter time step is used (e.g., $0.1 \mathrm{~s}$ instead of $1 \mathrm{~s}$ used in Fig. 4 simulations).

This simple example, together with similar simulations using different numbers of passive particles not shown here as well as results of the super-droplet approach available at the
University of Warsaw (Arabas et al., 2015), suggests that the simple scheme (Eq. 10) (and its extension into a threedimensional framework) should be used in the Lagrangian microphysics. Hence, such a scheme is used in all superdroplet simulations presented in this paper.

\subsection{Coupling thermodynamic Eulerian and Lagrangian fields}

The overall strategy for the time integration of the coupled Eularian and Lagrangian components of the model thermodynamics is to advance the temperature and moisture fields using Eq. (6) first, then to transport Lagrangian super-droplets using Eqs. (7) and (8), and finally to calculate condensation or evaporation of cloud droplets according to Eq. (4), with the condensation or evaporation providing temperature and moisture tendencies calculated from Eq. (3) in each grid cell. These tendencies are applied in the next model time step. Condensation or evaporation of individual superdroplets require knowledge of the supersaturation that needs to be calculated from updated temperature and water vapor fields. The flow-resolved supersaturation field can be supplemented with the subgrid-scale fluctuations as in GA17. By the same token, the resolved flow used to transport superdroplets through the predictor-corrector scheme can be supplemented with the subgrid-scale velocity fluctuations estimated from the predicted subgrid-scale turbulent kinetic energy. These additions are not included in the initial formulation and testing of the Twomey super-droplets discussed in this paper, but will form an important component of the model application in the future.

There are two issues that need to be considered for the coupling between Eulerian and Lagrangian model components. The first one concerns spurious supersaturation fluctuations near cloud edges (see Grabowski and Morrison, 2008, and references therein). This problem is particularly serious when the Twomey activation is used as illustrated later in the paper because of the direct link between the local supersaturation and the concentration of activated cloud droplets. Specifically, numerical overshoots of the supersaturation lead to an immediate activation of new cloud droplets. In contrast, when deliquescence and droplet activation are explicitly considered in the traditional super-droplet method, these transient overshoots may have a smaller impact on the droplet activation. This is one of the conclusions of the Hoffmann (2016) study, also confirmed by simulations discussed in this paper. Grabowski and Morrison (2008) developed a relatively simple method to cope with this problem for the case of a double-moment Eulerian microphysics scheme and suggested how it can be extended to the bin microphysics. We apply the Grabowski and Morrison (2008) methodology to the super-droplet simulations as discussed below.

The second issue concerns the interpolation of the thermodynamic fields to the super-droplet position. Shima et al. (2009) (see Sect. 5.1.2), Riechelmann et al. (2012) 
(Sect. 2.2.3), and Miroslaw Andrejczuk (personal communication, 2017) interpolate the potential temperature and water vapor mixing ratio and then derive the local supersaturation. Such an approach is not appropriate due to the nonlinear relationship between the supersaturation and the potential temperature. Interpolating the supersaturation would be more appropriate. However, supersaturation interpolation brings conceptual issues similar to those concerning super-droplet transport: if a single super-droplet represents a large ensemble of real cloud droplets, should growth of the ensemble be represented using the grid-averaged conditions? Moreover, one-dimensional tests with a stationary cloud-environment interface show that the supersaturation interpolation results in a gradual erosion of the cloud edge. This is because supersaturation interpolation between a cloudy grid cell near the cloud edge and a subsaturated cell outside the cloud results in subsaturated conditions for super-droplets located near the cell boundary leading to their evaporation. In contrast, applying the mean supersaturation maintains the steady conditions near the motionless cloud-environment interface. Moreover, applying the Grabowski and Morrison (2008) methodology to cope with the spurious cloud-edge supersaturation discussed below becomes cumbersome (if not impossible) when the supersaturation interpolation to the super-droplet position is used. Overall, our tests, similar to those discussed in the next section, suggest that the impact of supersaturation interpolation in a rising thermal simulations is small, and thus we decided to proceed with the simpler and computationally more efficient method of applying the grid-cell supersaturation to growth and evaporation of all super-droplets within a given grid cell.

\subsection{Avoiding spurious cloud-edge supersaturations}

The key aspect of the Grabowski and Morrison (2008) (GM08 hereinafter) method is to rely on the prediction of the absolute supersaturation (the difference between the water vapor mixing ratio and its saturated value) and to locally adjust the water vapor, cloud water, and temperature to maintain the predicted absolute supersaturation. This is in the spirit of Grabowski (1989), who used the temperature and supersaturation as main model variables and diagnosed the water vapor mixing ratio. Such a method results in a physically consistent supersaturation field but does not conserve water. GM08 circumvent this problem and apply the approach to the Eulerian double-moment cloud microphysics (i.e., predicting number and mass mixing ratios of the cloud water field). They also suggest how this approach can be used in the bin scheme (see Sect. 4 therein). Here we explain how this method is used with Twomey super-droplets.

The crux of the method is to calculate the amount of cloud water $\epsilon$ that needs to condense or evaporate to ensure that the predicted potential temperature and water vapor mixing ratio fields give the absolute supersaturation that agrees with the predicted one. Thus, in addition to the prediction of the potential temperature and water vapor mixing ratio, the scheme predicts the evolution of the absolute supersaturation (see Eq. A8 in Morrison and Grabowski, 2008, and Eq. 4 in GM08). Once the amount of cloud water involved in the adjustment is calculated as in Eq. (7) of GM08, one needs to decide how that amount is distributed among superdroplets present within a given grid cell. Following GM08, the amount of cloud water $\epsilon$ that needs to be distributed among $N$ super-droplets from a given cell is calculated as

$$
\begin{aligned}
& \epsilon=\sum_{i=1}^{N} \epsilon_{i}, \\
& \epsilon_{i}=\frac{\epsilon}{\beta \tau_{i}}, \text { where } \beta=\sum_{k=1}^{N} \frac{1}{\tau_{k}},
\end{aligned}
$$

where $\tau_{i}=\left(4 \pi D_{\mathrm{v}} n_{i} r_{i}\right)^{-1}$ is the phase relaxation timescale for the $i$ th super-droplet ( $n_{i}$ is the concentration of droplets $i$ th super-droplet represents); see Eq. (A5) in Morrison and Grabowski (2008). Knowing $\epsilon_{i}$, the radius of each superdroplet within a given grid cell is subsequently modified, keeping the multiplicity unchanged.

\section{Example of application: two-dimensional moist thermal simulations}

The scheme described above has been merged with the EULAG model (e.g., Prusa et al., 2008, www2.mmm.ucar.edu/ eulag/) and its simplified version referred to as babyEULAG (Grabowski, 2014, 2015). Here we present results from the babyEULAG model as it is simpler and thus more convenient for the scheme testing and improvement. The University of Warsaw Lagrangian Cloud Model (UWLCM) briefly described in the next section is used in the comparison. The Lagrangian approach applied in the UWLCM is referred to as the traditional super-droplet method in the discussion below. Both the babyEULAG model and the UWLCM apply the implicit large-eddy simulation approach, that is, without modeling of the unresolved subgrid-scale transport (see references to other studies applying this method in Grabowski, 2014; Pedersen et al., 2016).

\subsection{The University of Warsaw Lagrangian Cloud Model, UWLCM}

The UWLCM is an open-source software for twodimensional and three-dimensional modeling of clouds with super-droplet or bulk microphysics. Advection of the Eulerian fields is done using the libmpdata++ (Jaruga et al., 2015) implementation of the MPDATA algorithm (Smolarkiewicz and Margolin, 1998). Cloud microphysics is modeled using the libcloudph++ library (Arabas et al., 2015). Coupling between Eulerian and Lagrangian model components is done in the same way as in the Twomey model. Potential temperature and water vapor mixing ratio are not interpolated to the 
position of a super-droplet, but the same value is used for all droplets within a cell. The procedure for limiting spurious supersaturation, which was described in Sect. 2.6, is not used. The super-droplets are advected with a predictor-corrector method with velocities interpolated to super-droplet position using the "simple" scheme defined in Sect. 2.4. In the MPDATA algorithm used in simulations presented in this paper, variable-sign fields were handled using the "abs" option of the libmpdata++ library (see Sect. 3.1.5 and 3.4.1 in Jaruga et al., 2015). Advection of Eulerian fields and of super-droplets is done with a $\Delta t=1 \mathrm{~s}$ time step. Water condensation is done using 10 substeps per time step of advection, resulting in a $\Delta t=0.1 \mathrm{~s}$ time step for condensation. Details of the substepping procedure are discussed in Arabas et al. (2015).

Because UWLCM explicitly represents CCN deliquescence, a more detailed droplet growth equation is used (see Sect. 5.1.3 in Arabas et al., 2015). The $\kappa$-Köhler parametrization of aerosol hygroscopicity is used. We assume $\kappa=1.28$ for the sea salt aerosol used in this paper (see Table 1 in Petters and Kreidenweis, 2007). Super-droplets in the UWLCM typically have different multiplicities and only the initial number of super-droplets per grid cell is prescribed. The super-droplet initialization scheme is the same as in Dziekan and Pawlowska (2017) (the "constant SD" type of simulation described in Sect. 2 therein).

\subsection{Setup of moist thermal simulations}

Rising moist thermal simulations follow Grabowski and Clark (1991, 1993) with small modifications. The environmental profiles are taken as constant stability $\mathrm{d} \ln \Theta_{\mathrm{v}} / \mathrm{d} z=$ $1.3 \times 10^{-5} \mathrm{~m}^{-1}$ for the temperature $\left(\Theta_{\mathrm{v}}\right.$ is the virtual potential temperature; the potential-temperature-based stability was used in Grabowski and Clark, 1991, 1993) and constant relative humidity of $20 \%$. Surface temperature and pressure are taken as $283 \mathrm{~K}$ and $850 \mathrm{hPa}$. Note that the $\Theta_{\mathrm{v}}$-based stability profile requires an iterative procedure when moving upwards from the surface because the temperature, moisture, and pressure (the latter resulting from the hydrostatic balance) have all to be adjusted to give stability and relative humidity profiles exactly as specified above. The circular moisture perturbation is introduced in the middle of the $3.6 \mathrm{~km}$ horizontal domain, with the center located at the $800 \mathrm{~m}$ height. The vertical extent of the domain is $2.4 \mathrm{~km}$. The air inside the $250 \mathrm{~m}$ perturbation radius ( $200 \mathrm{~m}$ was used in Grabowski and Clark, 1991, 1993) is assumed to be saturated, and the relative humidity decreases to the environmental value as cosine squared over the $100 \mathrm{~m}$ radial distance. Uniform horizontal and vertical grid length of $20 \mathrm{~m}$ is used. A $1 \mathrm{~s}$ time step is used in simulations applying the babyEULAG model.

The average number of super-droplets per grid cell affects the amplitude of statistical fluctuations due to the transport of super-droplets across the Eulerian grid. Because of dif- ferent formulations of $\mathrm{CCN}$ activation, a direct match of the super-droplet number per grid cell between UWLCM and the Twomey scheme is impossible. In the Twomey scheme simulations, the number of $S_{\max }$ divisions considered was 50, 200, 1000 , and 4000 . The corresponding number of super-droplets per cloudy grid cell was around 40 for 50 divisions, $\sim 150$ for 200 divisions, $\sim 700$ for 1000 divisions, and $\sim 2700$ for $4000 S_{\max }$ divisions. The UWLCM simulations used in the comparison with the Twomey scheme simulations discussed in the next section applied 200 and 4000 super-droplets per grid cell. Only some of them became activated, and the averaged fraction of activated super-droplets (i.e., those with the radius larger than the critical radius) was around $45 \%$ for both cases.

\subsection{Comparison between UWLCM and the Twomey super-droplets}

When comparing results from the two models, one needs to keep in mind that microphysical schemes differ in some additional details. In particular, the UWLCM applies the $\kappa$-Köhler parametrization (Petters and Kreidenweis, 2007) to prescribe $\mathrm{CCN}$ activation characteristics whereas the Twomey scheme applies the N-S relationship derived from activation calculations applying $\mathrm{CCN}$ chemical composition information (i.e., as in Grabowski et al., 2011). Our tests with the adiabatic parcel model applying either the $\kappa$-Köhler parametrization used in UWLCM or the approach based on the CCN chemical composition show that difference of a couple of percentage points between droplet concentration predicted by the two methods for the same supersaturation is not unusual. Moreover, different droplet growth equations are used in the two schemes, although this factor does not affect the favorable comparison presented in Grabowski et al. (2011). Finally, the number of SDs representing activated $\mathrm{CCN}$ used in both models is not exactly the same as explained before.

Figures 5 and 6 show spatial distributions of the water vapor and cloud water mixing ratios for the two simulations, that is, using either the Twomey super-droplets with the babyEULAG model (Fig. 5) or the traditional super-droplets with UWLCM model (Fig. 6). Both simulations apply a similar number of super-droplets per grid cell (4000 aerosol sizes in UWLCM and 4000 divisions in the Twomey simulation). Overall, the transition of the initial circular perturbation to a cloudy rising vortex pair proceeds similarly in the two models. The most obvious difference comes from the development of instabilities near the thermal top. These instabilities are forced by fluctuations of thermodynamic fields (and thus cloud buoyancy) that result from a finite number of super-droplets in each grid cell. As discussed in Grabowski and Clark (1991, 1993), these cloud-environment instabilities represent a combination of Rayleigh-Taylor and KelvinHelmholtz instabilities occurring in a complex geometry near the thermal leading edge. The spatial scale of the instabil- 


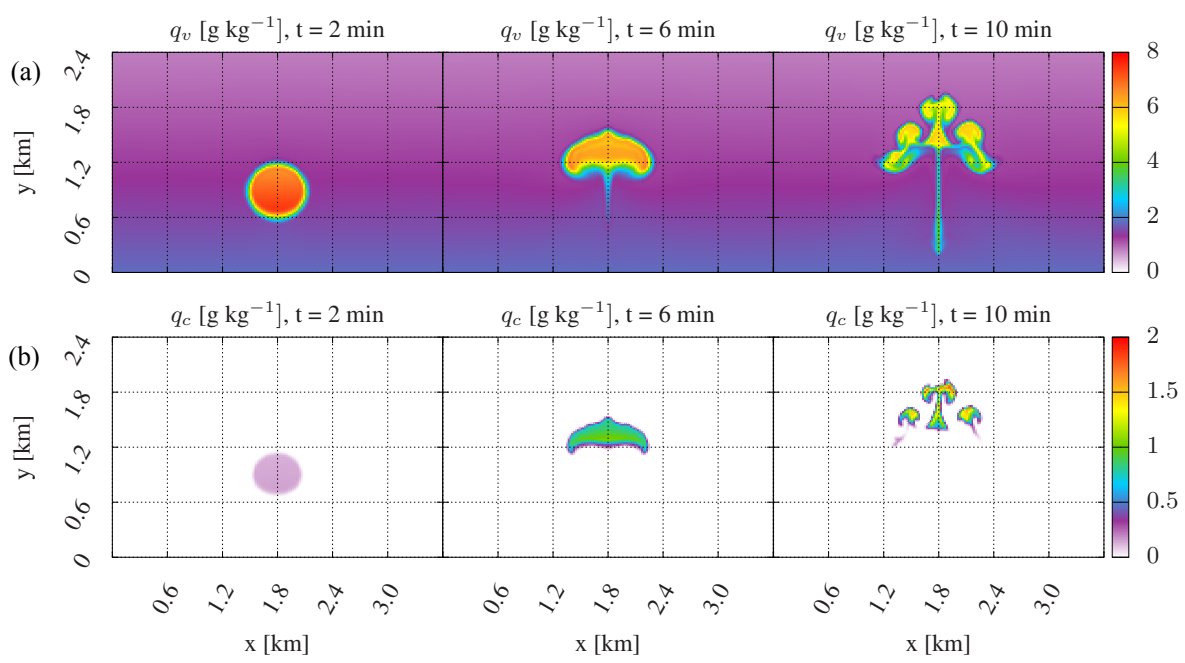

Figure 5. Distribution of (a) the water vapor and (b) the cloud water mixing ratios at 2, 6 and 10 min for the Twomey super-droplet scheme in the rising thermal simulation.
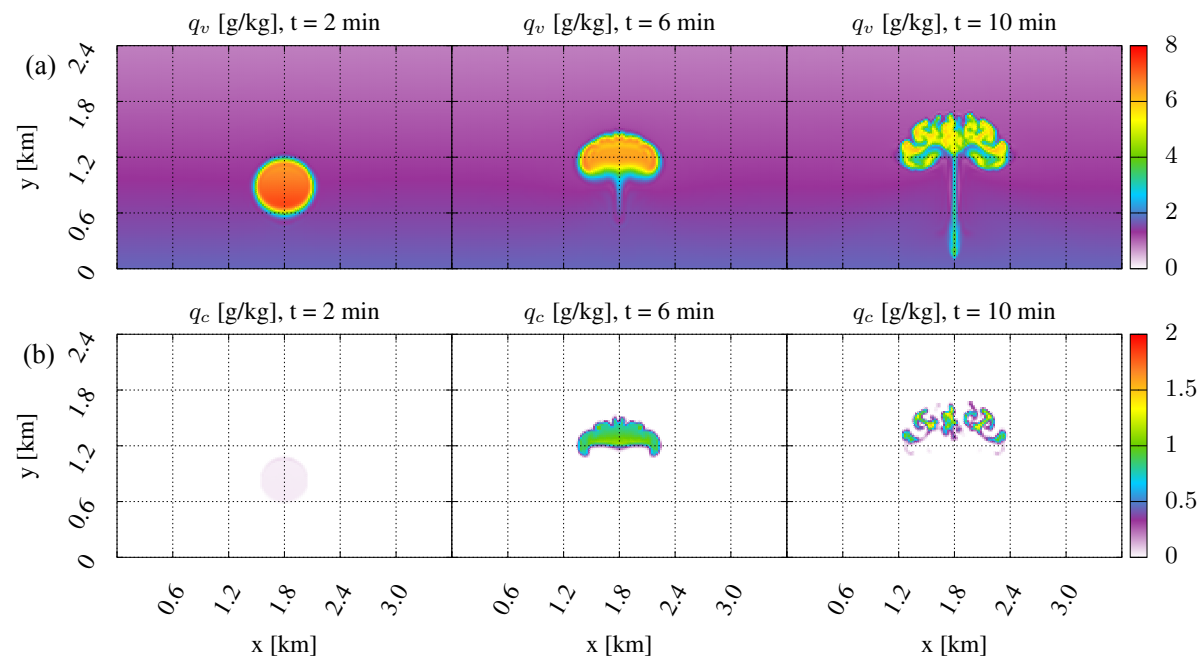

Figure 6. As Fig. 5, but for the UWLCM model.

ity depends on the depth of the shear that develops near the cloud-environment interface as the thermal pushes upwards (see Sect. 4b in Grabowski and Clark, 1991). The specific realization of the instability pattern changes with the number of super-droplets used in the simulation, and with the selection of random numbers applied during positioning superdroplets on the Eulerian grid during activation. It follows that the direct comparison between the simulations is possible only before the development of the instabilities, say, up to the 6th minute of the simulation (i.e., middle panels in Figs. 5 and 6).

A more detailed comparison between the two simulations is facilitated by applying two different statistical measures. The first one involves conditional sampling of various fields across the thermal, including points with the cloud water mixing ratio exceeding a threshold of $0.1 \mathrm{~g} \mathrm{~kg}^{-1}$. A smaller threshold allows incorporation of more significant fraction of points from the thermal edge that are affected by the Eulerian model numerics. The statistics include the mean values of conditionally sampled fields and the standard deviations of the spatial variability of a given field across the thermal. The second measure is the time evolution of various quantities at the center of mass of the cloud water field, that is, at the height of $z_{\mathrm{cm}}=\int z q_{\mathrm{c}} \mathrm{d} s / \int q_{\mathrm{c}} \mathrm{d} s$ (where $q_{\mathrm{c}}$ depicts the cloud water mixing ratio and the integral is over the entire computational domain) and a similar expression for the horizontal position $x_{\mathrm{cm}}$.

Figure 7 compares evolution of the supersaturation field conditionally sampled over the rising thermal for UWLCM with 200 and 4000 super-droplets per grid cell and the 

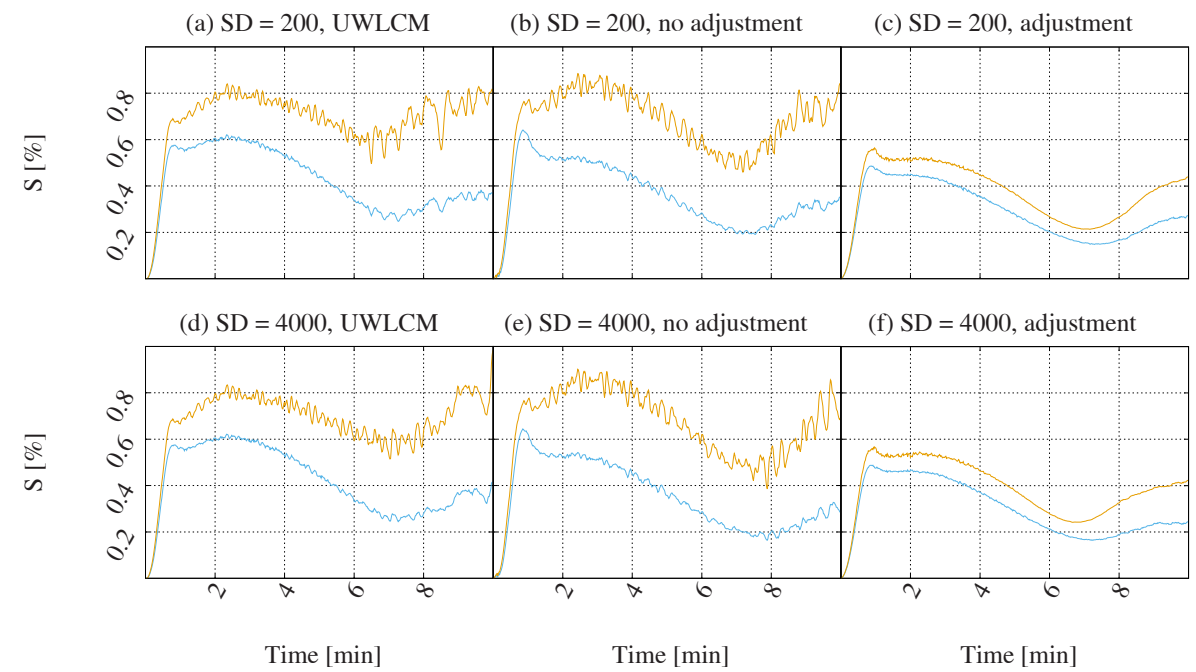

Figure 7. Comparison of the mean supersaturation averaged over the cloudy points for UWLCM (a, d) and the Twomey super-droplet scheme without $(\mathbf{b}, \mathbf{e})$ and with $(\mathbf{c}, \mathbf{f})$ the adjustment to avoid unphysical cloud-edge supersaturation fluctuations. The blue and brown lines represent the mean and the mean plus the standard deviation of the spatial distribution. Panels (a-c) and (d-f) are for simulations with 200 and 4000 super-droplets (UWLCM) or number of divisions (Twomey scheme). Data are plotted at every time step of the fluid flow model.

Twomey approach with 200 and $4000 S_{\max }$ divisions. The Twomey results either include or exclude (marked adjust and noadjust in the figure) temperature and moisture adjustment as described in Sect. 2.6. The figure shows that application of the adjustment scheme is critical for maintaining a physically consistent supersaturation field as the supersaturations are significantly higher without the adjustment. The physical consistency is measured by comparing the supersaturation predicted locally with the quasi-equilibrium supersaturation, that is, the supersaturation resulting from a balance between production due to the updraft and removal due to condensation (Squires, 1952; Politovich and Cooper, 1988). Except for the initial first minute when droplets are small, the supersaturation predicted by the model agrees well with the quasi-equilibrium supersaturation (not shown). An important point is that simulations with 200 divisions (upper panels) differ little from simulations applying 4000 divisions (lower panels) until different flow realizations in the final few minutes cause the divergence. The agreement suggests that about a hundred super-droplet per grid cell is sufficient to obtain statistics that change little with further increase of the superdroplet number. The reduction of both the standard deviation and the amplitude of the fluctuations when the adjustment scheme is applied is also apparent. The corresponding results from UWLCM simulations show that the mean supersaturation evolution is similar to those for the Twomey simulations without adjustment as one might expect.

Figure 8 shows statistics of the droplet concentration in the format similar to Fig. 7. As expected, the mean concentration is higher when adjustment is not used in the Twomey approach. The mean concentration slowly decreases in time because of the air expansion due to rise of the thermal. For
Twomey simulations with $200 S_{\max }$ divisions, the mean concentration at minute 2 is around $77 \mathrm{~cm}^{-3}$ and the standard deviation of the spatial distribution is around $5 \mathrm{~cm}^{-3}$ in simulation with the adjustment versus $85 \mathrm{~cm}^{-3}$ and higher standard deviation without the adjustment. The mean concentration at 2 min decreases to around $72 \mathrm{~cm}^{-3}$ for the 4000 division simulations. For the UWLCM, the mean concentration at minute 2 is around $75 \mathrm{~cm}^{-3}$ and the standard deviation of the spatial distribution is around $9 \mathrm{~cm}^{-3}$. The similarity of the mean concentration between the Twomey super-droplets with adjustment and UWLCM documents the limited impact of the cloud-edge supersaturation fluctuations when details of the $\mathrm{CCN}$ activation are resolved in the original super-droplet approach. Larger standard deviation of the spatial distribution is likely because of the variable multiplicity attribute among original super-droplets in the UWLCM model.

Figures 9 and 10 compare various statistics between the Twomey scheme and UWLCM at the center of mass of the cloud water field with 200 and 4000 super-droplets (UWLCM) or $S_{\max }$ divisions (Twomey scheme), 200 in Fig. 9, and 4000 in Fig. 10. The data are plotted at every model time step. Microphysical properties such as the droplet concentration, mean radius, and the spectral width show oscillations that are reduced with the increased number of super-droplets per grid cell. It is important to note that center of mass is calculated on the Eulerian grid, that is, it jumps from one grid box to another as the thermal moves upwards. The period of the oscillations in Figs. 9 and 10, about $10 \mathrm{~s}$, matches approximately the propagation of the center of mass over the grid as the updraft velocity is about $2 \mathrm{~m} \mathrm{~s}^{-1}$ and the grid length is $20 \mathrm{~m}$. The amplitude of the oscillations can be estimated by comparing the original evolution (i.e., the 

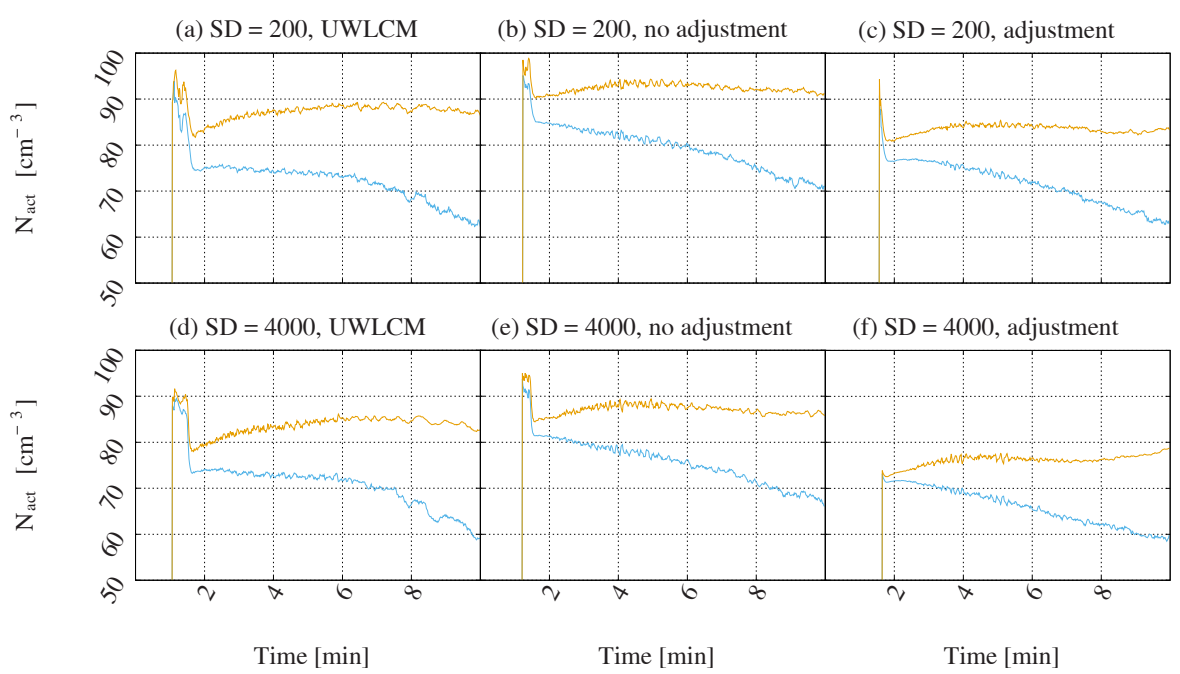

Figure 8. As in Fig. 7, but for the droplet concentration $N_{\text {act }}$.
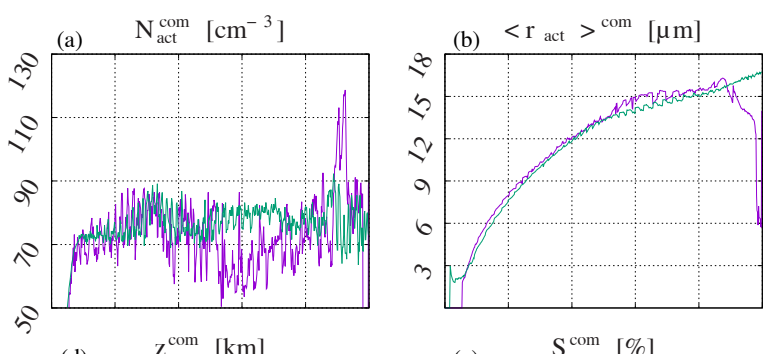

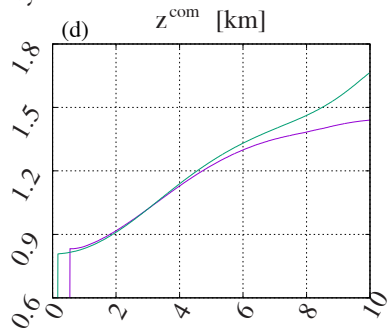

Time [min]

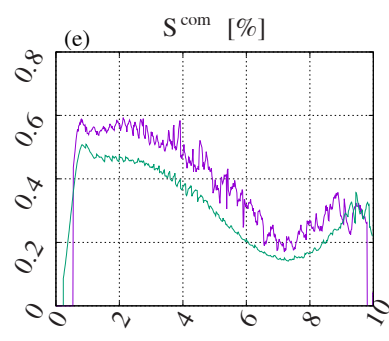

Time $[\mathrm{min}]$
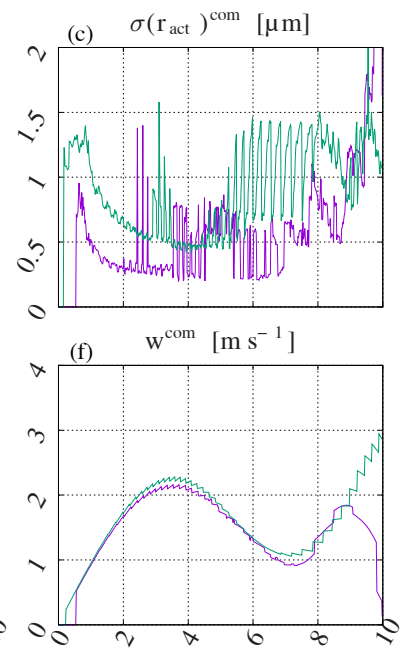

Time [min]

Figure 9. Evolution of various parameters at the center of mass of the cloud water in the rising thermal simulations applying 200 superdroplets (UWLCM; purple lines) or 200 divisions (Twomey scheme with adjustment; green lines). The panels show (a) droplet concentration, (b) droplet mean radius, (c) spectral width of the droplet size distribution, (d) height of the center of mass, (e) supersaturation, and (f) vertical velocity.

one shown in Figs. 9 and 10) and the evolution sufficiently smoothed in time so the oscillations are removed. In the case of the droplet concentration for the Twomey simulations, the amplitude decreases from 6.0, 2.0, 1.1, and $0.6 \mathrm{~cm}^{-3}$ for the number of divisions increasing from 50,200,1000, and 4000. This is roughly the expected scaling, that is, along the square root of the number of super-droplets that increases from around 40 to 2700 for the number of divisions increasing from 50 to 4000 . As mentioned before, the direct comparison between various simulations is only possible up to about the 6th minute as different flow evolutions make results impossible to compare at later times. Except for the oscillation amplitude, the results for different number of $S_{\max }$ divisions compare well for the Twomey super-droplets.

The differences between Figs. 9 and 10 are consistent with the differences between conditionally averaged statistics. For instance, droplet concentrations fluctuate between 60 and $80 \mathrm{~cm}^{-3}$ for Twomey super-droplets with adjustment and $200 S_{\max }$ divisions and UWLCM with 200 super-droplets per grid cell. The evolution of the center of mass height is very similar in Twomey and UWLCM simulations. The mean radius is close to $15 \mu \mathrm{m}$ at minute 6 for both the Twomey and UWLCM. Droplet concentration fluctuations are larger in UWLCM arguably because of the way CCN is sampled 

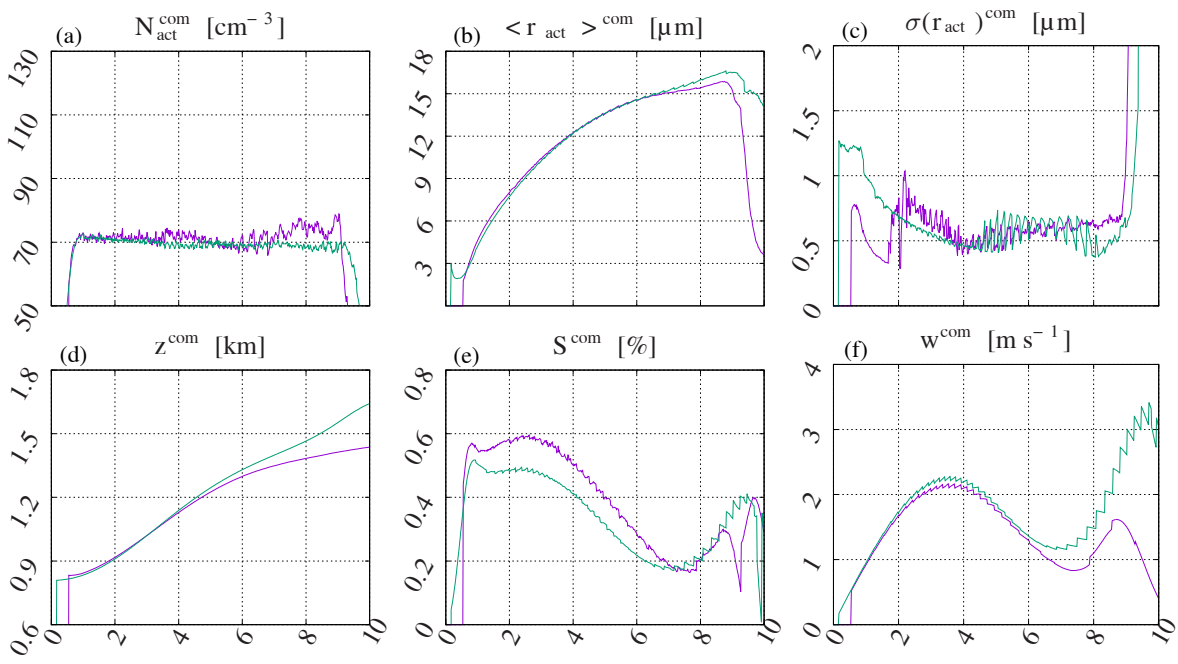

Time $[\mathrm{min}]$

Time $[\mathrm{min}]$

Time $[\mathrm{min}]$

Figure 10. As Fig. 9, but for 4000 super-droplets (UWLCM) or 4000 divisions (Twomey scheme).

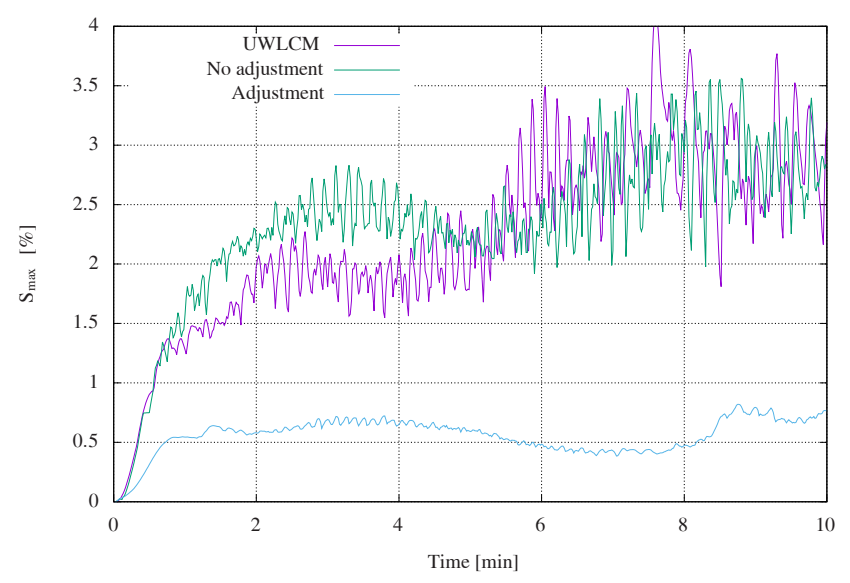

Figure 11. Evolution of the maximum supersaturation in the domain for UWLCM simulations (purple line) and Twomey scheme with (blue line) and without (green line) adjustment for unphysical supersaturation fluctuations. Data are plotted at every model time step with 200 super-droplets in UWLCM and 200 division in the model with the Twomey activation.

when super-droplets are created, that is, with different multiplicity parameters that increase the oscillation amplitude. The evolution of the vertical velocity at the cloud water center of mass increases in both simulations up to about $3.5 \mathrm{~min}$ and decreases thereafter (the evolutions after minute 6 cannot be compared due to different flow realizations as already explained). The vertical velocity maxima around $3.5 \mathrm{~min}$ are similar.

As a final element of the comparison, we show in Fig. 11 evolutions of the maximum supersaturation in the computational domain for simulations with the Twomey super- droplets with and without the adjustment to limit unphysical cloud-edge supersaturations and for the UWLCM. The simulations apply similar number of super-droplets per grid cell (200 divisions in the Twomey super-droplet simulations and 200 samples of the CCN distribution in the UWLCM simulation). The maximum supersaturations occur at different spatial locations near the cloud edge as the cloudenvironment interface moves across the Eulerian grid. This is why large fluctuations in the UWLCM simulation impact the mean droplet concentration to a smaller degree than in the Twomey approach. In a nutshell, $\mathrm{CCN}$ has no time to respond to these fluctuations when deliquescence is explicitly calculated by the model. In contrast, Twomey activation immediately adds new droplets when supersaturation fluctuations take place. These additional droplets can evaporate in subsequent time steps, but some survive and lead to the increased mean droplet concentration as documented in Fig. 8. It follows that the adjustment is the key element of the Twomey super-droplets, but is less significant for the traditional superdroplet approach; see Hoffmann (2016).

In summary, we believe that simple tests presented in this section document the efficacy of the super-droplet approach with the Twomey activation. Unfortunately, we cannot provide a direct comparison of the computational effort between the two approaches because the two models run on different computer systems. However, since the cloud covers about $2.5 \%$ of the two-dimensional computational domain, the Twomey scheme requires roughly 40 times less computational effort for simulations presented here (this estimate excludes the difference in the time steps used by both models). However, for a hypothetical three-dimensional simulation with a domain extending $3.6 \mathrm{~km}$ in the second horizontal direction, the volume of the initial spherical bubble with the same radius would only constitute about $0.1 \%$ of the com- 
putational domain volume. Thus, the computational effort in similar three-dimensional simulations would be about 3 orders of magnitude larger in UWLCM than in the babyEULAG with Twomey super-droplets. UWLCM makes up a lot of this difference by applying modern software engineering techniques including parallel processing and application of graphics processing units; see Arabas et al. (2015). It is important to note, however, that parallelization of the numerical model applying Twomey super-droplets requires a different strategy than the domain decomposition approach typically applied in finite-difference numerical models. This is to avoid significant load imbalances between subdomains featuring cloudy grid cells (i.e., with super-droplets) and those that are cloud-free. It remains to be seen whether such a different parallelization will lead to problems due to an increased communication.

\section{Conclusions}

This paper discusses technical details of a novel Lagrangian condensation scheme to model nonprecipitating warm (icefree) clouds. The idea is to use Lagrangian point particles ("super-droplets" following the nomenclature introduced by Shima et al., 2009), rather than continuous medium variables such as number or mass mixing ratios, to represent condensed cloud water. Previous studies applying such methodology (e.g., Andrejczuk et al., 2008, 2010; Shima et al., 2009; Riechelmann et al., 2012; Arabas et al., 2015; Hoffmann et al., 2015) demonstrate significant advantages of the super-droplet method, such as reduced numerical diffusion, formulation of the governing equations from first principles, and straightforward application of suitable statistical techniques to represent unresolved subgrid-scale variability as in GA17. However, in previous applications of the Lagrangian microphysics, the super-droplets outside clouds represent unactivated CCN that become activated upon entering a cloud and can further grow through diffusional and collisional processes. Thus, the super-droplets fill the entire computational domain and need to be transported even if they exist far away from a cloud and do not affect cloud processes. The original methodology allows for the detailed study of not only effects of CCN on cloud microphysics and dynamics, but also CCN processing by a cloud. When applying the superdroplet method to problems where CCN processing is of secondary importance (e.g., the impact of entrainment on the spectrum of cloud droplets), a simpler and more computationally efficient approach can be used. The idea is to create super-droplets only when $\mathrm{CCN}$ is activated and to remove them when a complete evaporation (i.e., $\mathrm{CCN}$ de-activation) takes place. Thus, no super-droplet exists outside a cloud and a significantly smaller number of super-droplets need to be followed in space and time when compared to the traditional super-droplet scheme with the same number of superdroplets per grid cell. The new super-droplet approach is possible by applying the Twomey activation method where the local supersaturation dictates the concentration of cloud droplets (and thus the number of the super-droplets) that need to be present inside a cloudy volume. Twomey activation excludes details of the $\mathrm{CCN}$ deliquescence and activation, and super-droplets simply disappear when a complete evaporation of cloud droplets occurs. Twomey activation is often used in Eulerian bulk (e.g., Morrison and Grabowski, 2007, 2008) and bin microphysics schemes (e.g., Grabowski et al., 2011; Wyszogrodzki et al., 2011). Moreover, simulation of the $\mathrm{CCN}$ deliquescence requires short time steps and avoiding it with the Twomey activation provides additional computational advantage. As mentioned previously, applying Twomey activation to create cloud droplets bears similarities to the way ice particles are initiated in a Lagrangian model targeting ice processes (e.g., Sölch and Kärcher, 2010).

We apply the traditional Lagrangian super-droplet model, the University of Warsaw Lagrangian Cloud Model (UWLCM; Arabas et al., 2015; Jaruga et al., 2015) and compare results from UWLCM and the novel Twomey superdroplet method. The simulations apply an idealized setup of a moist thermal rising in a stratified environment (Grabowski and Clark, 1991, 1993). Overall, the comparison demonstrates the efficacy of the new approach as simulation results differ little between UWLCM and the new scheme. This is consistent with adiabatic parcel results discussed in Grabowski et al. (2011) that - away from the cloud base show good agreement between cloud properties simulated applying a scheme with Twomey activation and a scheme where details of the $\mathrm{CCN}$ deliquescence are modeled explicitly. The results presented here show that avoiding spurious cloud-edge supersaturation fluctuations is essential with the Twomey activation. This is because these fluctuations immediately translate into unphysical droplet concentrations that affect subsequent evolution of cloud microphysical properties. In contrast, these fluctuations that are highly transient in space and time seem to have small impact on simulations using the original super-droplet method, in agreement with results discussed in Hoffmann (2016).

As noted in Clark (1974), Morrison and Grabowski (2008), and Grabowski and Jarecka (2015), modeling cloud base activation in the Eulerian cloud model requires high vertical resolution to resolve cloud base supersaturation maximum, say, of the order of $10 \mathrm{~m}$. The same is true for the Lagrangian super-droplets. In the case of lower vertical resolution (i.e., when the cloud base supersaturation maximum is poorly resolved), an activation parameterization can be used, for instance, linking the concentration of activated CCN to the strength of the updraft velocity (e.g., Abdul-Razzak et al., 1998; Abdul-Razzak and Ghan, 2000, among others). Such a parameterization can also be used with the methodology presented in this paper, for instance, in simulations of deep convection that only allow low vertical resolution. As deep convection requires incorporation of ice physics into the Lagrangian methodology, the possibility of apply- 
ing an even simpler representation of super-droplet formation through the activation parameterization is appealing. Such a methodology will pave the way for applications of the Lagrangian methodology beyond high-spatial-resolution largeeddy simulation today to the cloud-resolving (convectionpermitting) weather and climate simulation of the future. We plan to include such developments to the Twomey superdroplet scheme presented here, together with the inclusion of the collision-coalescence that will be the focus of future scheme expansions. These developments will be reported in forthcoming publications.

Code availability. The simulations with the Twomey activation of super-droplets were done using the bE_SDs v0.1 model, available at https://doi.org/10.5281/zenodo.1050260. The UWLCM v0.2 code is available at https://doi.org/10.5281/zenodo.1043909. 


\section{Appendix A: Subgrid-scale divergence of the simple interpolation scheme}

Figure A1 shows a grid cell with a subgrid volume of dimensions $\Delta \alpha$ and $\Delta \gamma$ with velocities $u_{1}, u_{2}, w_{1}$, and $w_{2}$ on the volume boundaries. Using the simple interpolation scheme (10) and symbols defined in Fig. A1, the horizontal velocities $u_{1}$ and $u_{2}$ are given by

$u_{1}=\left(\alpha-\frac{\Delta \alpha}{2}\right) u_{i+\frac{1}{2}, k}+\left(1-\alpha+\frac{\Delta \alpha}{2}\right) u_{i-\frac{1}{2}, k}$,

$u_{2}=\left(\alpha+\frac{\Delta \alpha}{2}\right) u_{i+\frac{1}{2}, k}+\left(1-\alpha-\frac{\Delta \alpha}{2}\right) u_{i-\frac{1}{2}, k}$,

and the vertical velocities $w_{1}$ and $w_{2}$ are as follows:

$w_{1}=\left(\gamma-\frac{\Delta \gamma}{2}\right) w_{i, k+\frac{1}{2}}+\left(1-\gamma+\frac{\Delta \gamma}{2}\right) w_{i, k-\frac{1}{2}}$,

$w_{2}=\left(\gamma+\frac{\Delta \gamma}{2}\right) w_{i, k+\frac{1}{2}}+\left(1-\gamma-\frac{\Delta \gamma}{2}\right) w_{i, k-\frac{1}{2}}$.

The divergence over the small volume (with dimensional extensions of $\Delta \alpha \Delta x$ and $\Delta \gamma \Delta z$ ) is then given by the following:

$\frac{u_{2}-u_{1}}{\Delta \alpha \Delta x}+\frac{w_{2}-w_{1}}{\Delta \gamma \Delta z}=$

$\frac{u_{i+\frac{1}{2}, k}-u_{i-\frac{1}{2}, k}}{\Delta x}+\frac{w_{i, k+\frac{1}{2}}-w_{i, k-\frac{1}{2}}}{\Delta z}=-\frac{w}{\rho} \frac{\partial \rho}{\partial z}$.

It follows that the divergence over the subgrid volume is exactly the same as over the grid cell volume. This is the key feature of the simple interpolation scheme (Eq. 10) because it allows transport of super-droplets in a physically consistent manner as documented in the passive particle advection tests (see Fig. 4).

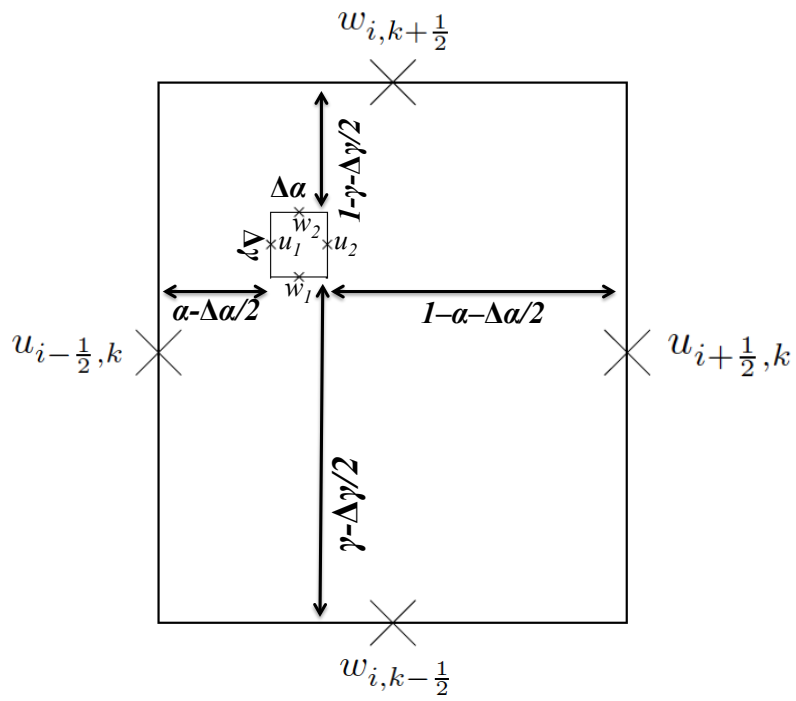

Figure A1. Interpolation of particle-advecting velocities from the grid cell (large rectangle) into a subgrid-scale volume (small rectangle) applying the simple interpolation scheme (Fig. 3). 
Author contributions. WWG developed the Twomey activation scheme and completed simulations using it. PD completed UWLCM simulations and created intercomparison figures. All authors contributed to the design of numerical simulations and were involved in creating the paper.

Competing interests. The authors declare that they have no conflict of interest.

Acknowledgements. This work was partially supported by the Polish National Science Center (NCN) "POLONEZ 1" Grant 2015/19/P/ST10/02596 and by the US DOE ASR Grant DESC0016476. The POLONEZ 1 grant has received funding from the European Union's Horizon 2020 Research and Innovation Program under the Marie Sklodowska-Curie grant agreement 665778. Wojciech W. Grabowski acknowledges discussions with Dorota Jarecka during the early development of Twomey super-droplet scheme. Helpful conversations and suggestions from Shin-Ichiro Shima (U. of Hyogo, Kobe, Japan) and from Lian-Ping Wang (U. of Delaware, Newark, USA) are also acknowledged. UWLCM development has been possible through NCN grants 2010/01/N/ST10/01438, 2012/06/M/ST10/00434 and 2014/15/N/ST10/05143. The National Center for Atmospheric Research is sponsored by the National Science Foundation.

Edited by: Simon Unterstrasser

Reviewed by: Shin-ichiro Shima and one anonymous referee

\section{References}

Abdul-Razzak, H. and Ghan, S. J.: A parameterization of aerosol activation: 2. Multiple aerosol types, J. Geophys. Res.-Atmos., 105, 6837-6844, 2000.

Abdul-Razzak, H., Ghan, S. J., and Rivera-Carpio, C.: A parameterization of aerosol activation: 1 . Single aerosol type, J. Geophys. Res.-Atmos., 103, 6123-6131, 1998.

Andrejczuk, M., Reisner, J., Henson, B., Dubey, M., and Jeffery, C.: The potential impacts of pollution on a nondrizzling stratus deck: Does aerosol number matter more than type?, J. Geophys. Res.Atmos., 113, D19204, https://doi.org/10.1029/2007JD009445, 2008.

Andrejczuk, M., Grabowski, W., Reisner, J., and Gadian, A.: Cloudaerosol interactions for boundary layer stratocumulus in the Lagrangian Cloud Model, J. Geophys. Res.-Atmos., 115, D22214, https://doi.org/10.1029/2010JD014248, 2010.

Arabas, S., Jaruga, A., Pawlowska, H., and Grabowski, W. W.: libcloudph++ 1.0: a single-moment bulk, double-moment bulk, and particle-based warm-rain microphysics library in C++, Geosci. Model Dev., 8, 1677-1707, https://doi.org/10.5194/gmd-8-16772015, 2015.

Clark, T. L.: A study in cloud phase parameterization using the gamma distribution, J. Atmos. Sci., 31, 142-155, 1974.

Clark, T. L.: Numerical simulations with a three-dimensional cloud model: Lateral boundary condition experiments and multicellular severe storm simulations, J. Atmos. Sci., 36, 2191-2215, 1979.
Dziekan, P. and Pawlowska, H.: Stochastic coalescence in Lagrangian cloud microphysics, Atmos. Chem. Phys., 17, 1350913520, https://doi.org/10.5194/acp-17-13509-2017, 2017.

Grabowski, W. W.: Numerical experiments on the dynamics of the cloud-environment interface: Small cumulus in a shear-free environment, J. Atmos. Sci., 46, 3513-3541, 1989.

Grabowski, W. W.: Extracting microphysical impacts in large-eddy simulations of shallow convection, J. Atmos. Sci., 71, 44934499, 2014.

Grabowski, W. W.: Untangling microphysical impacts on deep convection applying a novel modeling methodology, J. Atmos. Sci., 72, 2446-2464, 2015.

Grabowski, W. W. and Abade, G. C.: Broadening of Cloud Droplet Spectra through Eddy Hopping: Turbulent Adiabatic Parcel Simulations, J. Atmos. Sci., 74, 1485-1493, 2017.

Grabowski, W. W. and Clark, T. L.: Cloud-environment interface instability: Rising thermal calculations in two spatial dimensions, J. Atmos. Sci., 48, 527-546, 1991.

Grabowski, W. W. and Clark, T. L.: Cloud-environment interface instability: Part II: Extension to three spatial dimensions, J. Atmos. Sci., 50, 555-573, 1993.

Grabowski, W. W. and Jarecka, D.: Modeling condensation in shallow nonprecipitating convection, J. Atmos. Sci., 72, 4661-4679, 2015 .

Grabowski, W. W. and Morrison, H.: Toward the mitigation of spurious cloud-edge supersaturation in cloud models, Mon. Weather Rev., 136, 1224-1234, 2008.

Grabowski, W. W. and Smolarkiewicz, P. K.: A multiscale anelastic model for meteorological research, Mon. Weather Rev., 130, 939-956, 2002.

Grabowski, W. W., Andrejczuk, M., and Wang, L.-P.: Droplet growth in a bin warm-rain scheme with Twomey CCN activation, Atmos. Res., 99, 290-301, 2011.

Hoffmann, F.: The effect of spurious cloud edge supersaturations in Lagrangian cloud models: An analytical and numerical study, Mon. Weather Rev., 144, 107-118, 2016.

Hoffmann, F., Raasch, S., and Noh, Y.: Entrainment of aerosols and their activation in a shallow cumulus cloud studied with a coupled LCM-LES approach, Atmos. Res., 156, 43-57, 2015.

Jaruga, A., Arabas, S., Jarecka, D., Pawlowska, H., Smolarkiewicz, P. K., and Waruszewski, M.: libmpdata++ 1.0: a library of parallel MPDATA solvers for systems of generalised transport equations, Geosci. Model Dev., 8, 1005-1032, https://doi.org/10.5194/gmd-8-1005-2015, 2015.

Kessler, E.: Elementary theory of associations between atmospheric motions and distributions of water content, Mon. Weather Rev., 91, 13-27, 1963.

Klemp, J. B. and Wilhelmson, R. B.: The simulation of threedimensional convective storm dynamics, J. Atmos. Sci., 35, 1070-1096, 1978.

Kogan, Y. L.: The simulation of a convective cloud in a 3-D model with explicit microphysics, Part I: Model description and sensitivity experiments, J. Atmos. Sci., 48, 1160-1189, 1991.

Liu, J. and Orville, H.: Numerical modeling of precipitation and cloud shadow effects on mountain-induced cumuli, J. Atmos. Sci., 26, 1283-1298, 1969.

Morrison, H. and Grabowski, W. W.: Comparison of bulk and bin warm-rain microphysics models using a kinematic framework, J. Atmos. Sci., 64, 2839-2861, 2007. 
Morrison, H. and Grabowski, W. W.: Modeling supersaturation and subgrid-scale mixing with two-moment bulk warm microphysics, J. Atmos. Sci., 65, 792-812, 2008.

Murray, F. W.: Numerical models of a tropical cumulus cloud with bilateral and axial symmetry, Mon. Weather Rev., 98, 14-28, https://doi.org/10.1175/15200493(1970)098<0014:NMOATC>2.3.CO;2, 1970.

Pedersen, J. G., Malinowski, S. P., and Grabowski, W. W.: Resolution and domain-size sensitivity in implicit large-eddy simulation of the stratocumulus-topped boundary layer, J. Adv. Model. Earth Syst., 8, 885-903, 2016.

Petters, M. D. and Kreidenweis, S. M.: A single parameter representation of hygroscopic growth and cloud condensation nucleus activity, Atmos. Chem. Phys., 7, 1961-1971, https://doi.org/10.5194/acp-7-1961-2007, 2007.

Politovich, M. K. and Cooper, W. A.: Variability of the supersaturation in cumulus clouds, J. Atmos. Sci., 45, 1651-1664, 1988.

Prusa, J. M., Smolarkiewicz, P. K., and Wyszogrodzki, A. A.: EULAG, a computational model for multiscale flows, Comput. Fluids, 37, 1193-1207, 2008.

Riechelmann, T., Noh, Y., and Raasch, S.: A new method for largeeddy simulations of clouds with Lagrangian droplets including the effects of turbulent collision, New J. Phys., 14, 065008, https://doi.org/10.1088/1367-2630/14/6/065008, 2012.

Schlesinger, R. E.: A numerical model of deep moist convection: Part I, Comparative experiments for variable ambient moisture and wind shear, J. Atmos. Sci., 30, 835-856, 1973.

Shima, S.-I., Kusano, K., Kawano, A., Sugiyama, T., and Kawahara, S.: The super-droplet method for the numerical simulation of clouds and precipitation: A particle-based and probabilistic microphysics model coupled with a non-hydrostatic model, Q. J. Roy. Meteor. Soc., 135, 1307-1320, 2009.
Smolarkiewicz, P. K.: Multidimensional positive definite advection transport algorithm: an overview, Int. J. Numer. Methods Fluids, 50, 1123-1144, 2006.

Smolarkiewicz, P. K. and Margolin, L. G.: MPDATA: A finitedifference solver for geophysical flows, J. Comput. Phys., 140, 459-480, 1998.

Smolarkiewicz, P. K. and Margolin, L. O.: On forward-in-time differencing for fluids: extension to a curvilinear framework, Mon. Weather Rev., 121, 1847-1859, 1993.

Sölch, I. and Kärcher, B.: A large-eddy model for cirrus clouds with explicit aerosol and ice microphysics and Lagrangian ice particle tracking, Q. J. Roy. Meteor. Soc., 136, 2074-2093, 2010.

Squires, P.: The growth of cloud drops by condensation, I. General characteristics, Aust. J. Sci. Res., 5, 59-86, 1952.

Twomey, S.: The nuclei of natural cloud formation, part II: The supersaturation in natural clouds and the variation of cloud droplet concentration, Pure Appl. Geophys., 43, 243-249, 1959.

Unterstrasser, S. and Söslch, I.: Optimisation of the simulation particle number in a Lagrangian ice microphysical model, Geosci. Model Dev., 7, 695-709, https://doi.org/10.5194/gmd-7-6952014, 2014.

Unterstrasser, S., Hoffmann, F., and Lerch, M.: Collection/aggregation algorithms in Lagrangian cloud microphysical models: rigorous evaluation in box model simulations, Geosci. Model Dev., 10, 1521-1548, https://doi.org/10.5194/gmd-101521-2017, 2017.

Wyszogrodzki, A., Grabowski, W., and Wang, L.-P.: Activation of cloud droplets in bin-microphysics simulation of shallow convection, Acta Geophys., 59, 1168-1183, 2011. 